\title{
Research Resources
}

\section{A summary of new products and services for materials research...}

Imaging ToF SIMS for Ultrasensitive Surface Chemical Analysis: New imaging time-of-flight secondary ion mass spectrometer can give detailed chemical analysis with submicron spatial resolution and surface monolayer sensitivity. The sensitivity of the system allows a mass spectrum and four mass selected images to be acquired simultaneously from less than 10 femtograms of material corresponding to 0.004 atomic monolayers over an area $20 \mu \mathrm{m} \times 20 \mu \mathrm{m}$. The new system is designed to make it easier to study materials of low conductivity and also to improve the collection of data concerning trace impurities on surfaces, which can easily be sputtered away during the first few moments of normal SIMS analysis. The ToF SIMS allows analysis with a primary ion dose five orders of magnitude lower than a quadrupole spectrometer.

The system consists of a pulsed primary ion gun; sample handling facilities and a high stability stage; a high mass resolution, high mass range (over 8000 mass units) ToF mass spectrometer; and a framestore data system with single ion counting and nanosecond time resolution. Modular construction allows the instrument to be configured to individual needs and enables extra facilities to be added.

The primary ion gun uses a high brightness gallium liquid metal ion source and has a minimum probe size of 0.05 microns and a minimum pulse duration of 5 ns.System software enables acquisition of ion-induced SEM images as well as ToF SIMS images and mass spectra, with a realtime display of data as it is collected. The data system also enables a pulse repetition rate of up to $20 \mathrm{kHz}$ so that a full spectrum and image analysis can take less than 60 seconds.

The VG reflection mode Laser Microprobe Mass Analyzer can be added to the ToF SIMS at any time to combine two techniques - the speed of laser microprobe analysis and the sensitivity and precision of ToF SIMS - on the same instrument.

Two systems have already been installed in the United Kingdom, one for catalysis research at the Plastics and Petrochemical Division of Imperial Chemical Industries and one at the University of Manchester Institute of Science and Technology as part of a general surface analysis facility. VG Instruments, Inc., 300 Broad Street, Stamford, CT 06901; (203) 322-4546.

Technical Reports on Ion Implantation: Twelve technical reports presented at the Sixth International Conference on Ion Implantation (July 1986, Berkeley, CA) cover topics critical to semiconductor device production and the development of advanced

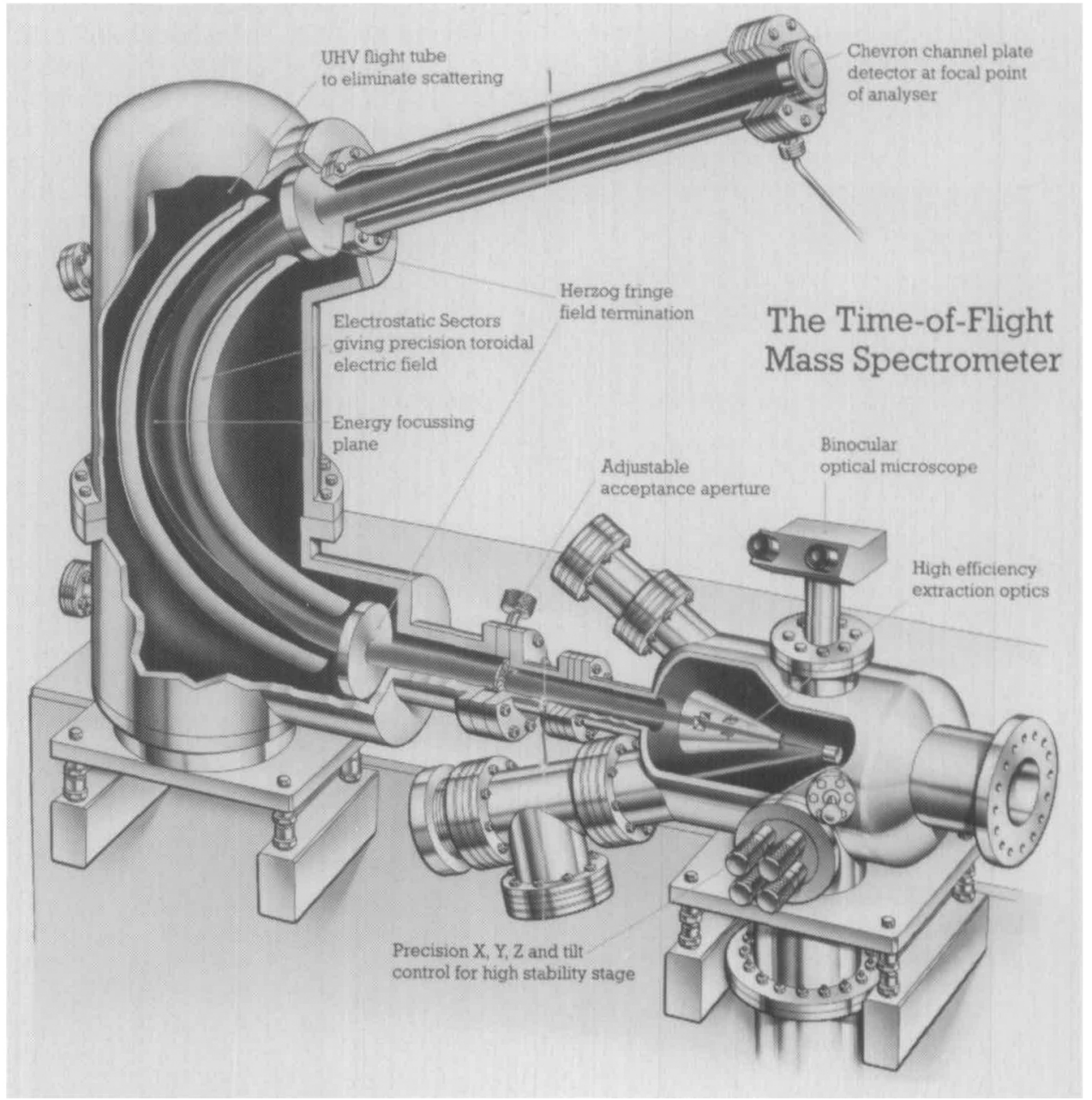

processing techniques, including: improving process yield and reliability, optimizing performance for automatic systems, controlling particulates, avoiding channeling effects, reducing wafer surface charging, maximizing dose uniformity, and forming boron-doped shallow junctions of low resistivity. Also detailed are the performance of Varian's VLSI compatible RTP system and the company's XP series high current ion implantation systems for processing wafers up to $200 \mathrm{~mm}$. Varian Associates, Semiconductor Equipment Group, G-105, 611 Hansen Way, Palo Alto, CA 94303; (800) 544-4636.

Sputter Deposition System: 8-page brochure describes the key features and benefits of the CVC 2800 Load Lock sputtering system, including capabilities of the color graphic software. Designed for high volume production, the CVC 2800 offers a high degree of process flexibility. It is the ideal system for many thin film deposition applications: R\&D, GaAs devices, hybrid devices, IC/LSI/VLSI devices, silicides, Schottky rectifiers, conventional bonding, tape automated bonding, thin film heads, solar cells, and liquid crystal displays. Also described are the operating principles of the Rotostrate ${ }^{\mathrm{TM}}$ transfer pivot arm designed to greatly reduce the level of particulates generated during processing. CVC Products, Inc., 525 Lee Road, P.O. Box 1886, Rochester, NY 14603; (716) 458-2550.

Excimer Laser System: XLR-100 Excimer Laser Research Center has multiple wavelength capability, submicron resolution, and computerized beam shaping and system operation. Optical, electrical, and mechanical systems have been combined to permit complex excimer laser experiments at 193, 248, or $308 \mathrm{~nm}$ to be performed conveniently and quickly with precise computer control. All results can be captured automatically on $35 \mathrm{~mm}$ film, or in real time by a video camera system or by the XLR-100 dedicated computer system. Unique optical design permits viewing of the specimen during excimer laser exposure. Leitz-Image Micro Systems Co., 900 Middlesex Turnpike, Building 8, Billerica, MA 01821; (617) 663-7070.

Continued 
Gridless Ion Source: Designed to be rugged and to minimize maintenance, the Mark II is a gridless ion source for production applications where wide area coverage, high curent density, and reactive gases are important. The Mark II supplies low energy ions over large areas at up to one ampere current over an energy range of $40-100 \mathrm{eV}$, or more. Completely compatible with processes using sustained op-

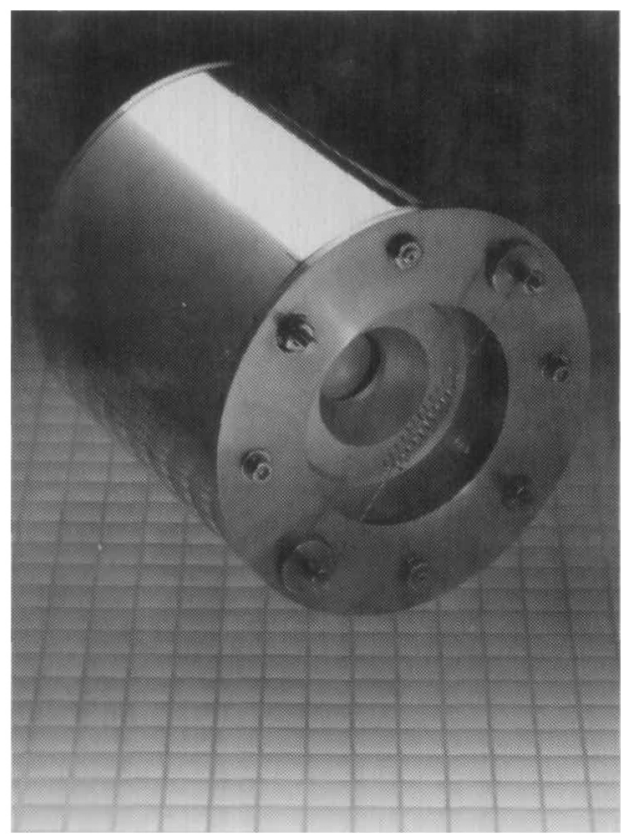

eration with reactive gases, it is used with oxygen and nitrogen for ion-assisted deposition of oxide and nitride films. The Mark II comes complete with a dedicated power supply. Commonwealth Scientific Corp., 500 Pendleton Street, Alexandria, VA 22314; (703) 548-0800.

Sputtering System for VLSI and ULSI: ECLIPSE $^{\mathrm{M}}$ cassette-to-casette sputter coater for VLSI and ULSI wafer fabrication promises true process isolation in a twotarget or three-target configuration. ECLIPSE addresses the issues of superior films, higher uptime, and full automation with a minimal clean room footprint, simple maintenance, and easy operation in the metallization of $125 \mathrm{~mm}, 150 \mathrm{~mm}$, and 200 $\mathrm{mm}$ wafers. Materials Research Corporation, Orangeburg, NY 10962; (914) 3594200 .

Ion Source for Surface Analysis: Adaptable ion source IQE-12/38 surface analysis component is an extractor type ion source with differential pumping on a dual lens system to facilitate spot size adjustments from $100 \mu \mathrm{m}$ to $1000 \mu \mathrm{m}$. It can operate at the low ion current densities required for static SIMS or provide the high beam currents necessary for rapid depth profiling or sputter cleaning. Argon is the normal operating gas, but the IQE-12/38 can operate with other inert gases and oxygen. The component is adaptable to most ultrahigh vacuum surface analysis instruments. Leybold-Heraeus Vacuum Products, Inc. (LAS Group), 5700 Mellon Road, Export, PA 15632; (412) 327-5700.

Crystal Growth Furnace: Model CG furnace can grow crystals by either Bridgman or Czochralski methods. The work chamber is available with either one or two heat zones, and the heat zone elements can be either graphite or refractory metal. A loading chamber, accessible from the front and separated from the heat zone by a stainless steel isolation valve, allows installation of a seed or removal of a grown crystal with the heat zone at temperature. The furnace can be interfaced to the purchaser's on-site computer system. CENTORR Associates, Route 28, Suncook, NH 03275; (603) 4859504.

Process Control Terminal for Ion Implantation: The PCT/XP process control terminal offers precise control of implants performed by serial ion implantation systems used on semiconductor devices. The PCT/EX, which controls and monitors all implant parameters, will minimize setup errors and enhance yield in semiconductor processing. Varian Associates, Inc., 611 Hansen Way, G-105; Palo Alto, CA 94303; (415) $493-4000$

Ion Implanter: Model 221 ion implanter operates at energies up to $90 \mathrm{kV}$ for smallscale $R \& D$ and for production. The ion source can be mounted on any face of the $20 \times 20 \times 20$ inch work chamber, and can be angled $\pm 30^{\circ}$ on any axis and has an integral isolator valve. The Model 221 offers a

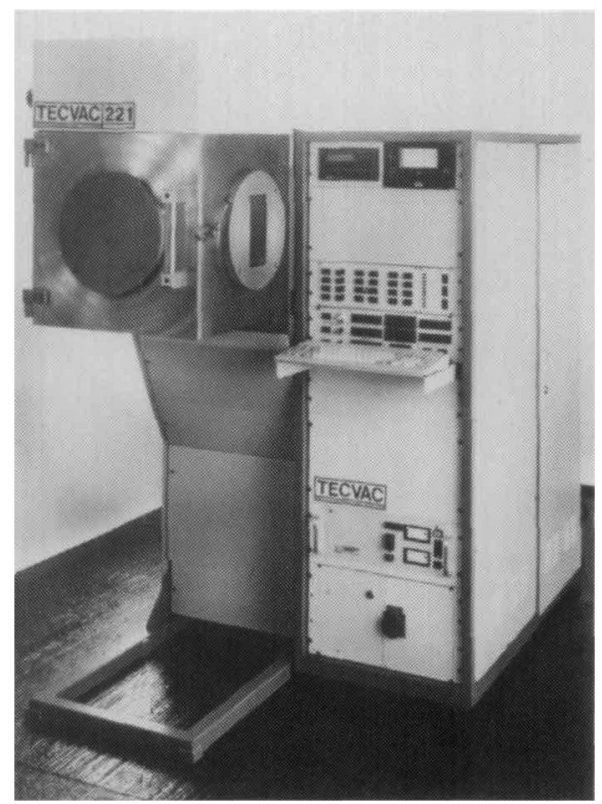

flexible modular batch implanter suitable for small-scale production or R\&D applications. Microscience, Forbes Business Center, 182 Forbes Road, Braintree, MA 02184; (617) 849-1952.
UHV Specimen Handling Work Station Tekscan IWS. Series Integrated Work Station offers a unique and versatile solution for specimen introduction and handling in UHV systems. The IWS incorporates a linear rack-and-pinion drive mounted on a

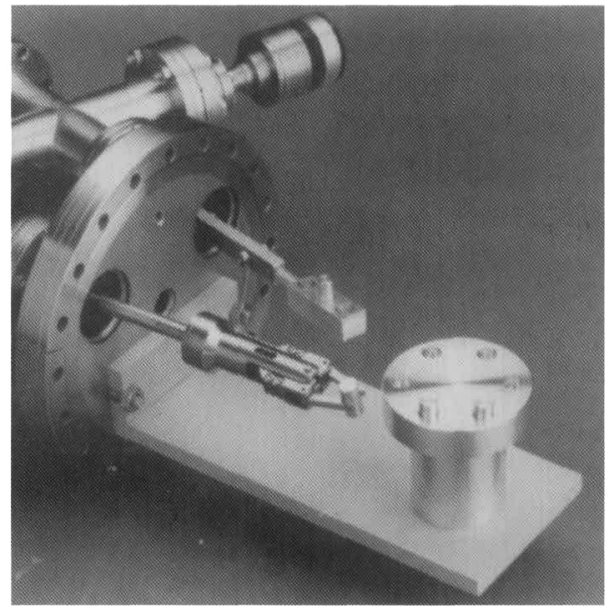

load-lock for sample entry and linear positioning, and a versatile sample handling system mounted onto a $200 \mathrm{~mm}$ or $150 \mathrm{~mm}$ diameter knife-edge flange for easy retrofit. Applications include in-UHV specimen preparation, positioning, and handling, and multi-sample handling. Optional extras include fracture stages, fiber-optic illuminator, and miniature TV camera and monitor for viewport surveillance. Microscience, Forbes Business Center, 182 Forbes Road, Braintree, MA 02184; (617) 849-1952.

Physics News in 1986: The 18th in a series of annual reviews of physics news, this $72-$ page report by the American Institute of Physics covers the following areas: astrophysics, chemical physics, condensed matter physics, crystallography, electron and atomic physics, elementary particle physics, fluid dynamics, geophysics, medical physics, nuclear physics, optics, physics applied to industry, physics education, plasma and fusion physics, polymer physics, vacuum physics, and the Nobel Prize in physics. Public Information Division, American Institute of Physics, 335 East 45th Street, New York, NY 10017; (212) 661-9404.

High-Energy Ion Implanter: Model 500 XP ion implanter can perform implants from $35 \mathrm{keV}$ to $1 \mathrm{MeV}$ for advanced semiconductor manufacturing applications. The system is unique in its ability to perform standard low-energy, medium-current implants as well as megavolt implantation. Varian Associates, Inc., 611 Hansen Way, G-105, Palo Alto, CA 94303; (800) 544-4636. 


\section{When Your Research Is So Important, Why Compromise On Sample Preparation?}

South Bay Technology has been providing the materials research community with superior sample preparation equipment for over 22 years. By designing and manufacturing our own equipment, we have built a reputation for uncompromising quality, precision and service. By listening to your needs, we have been able to meet your

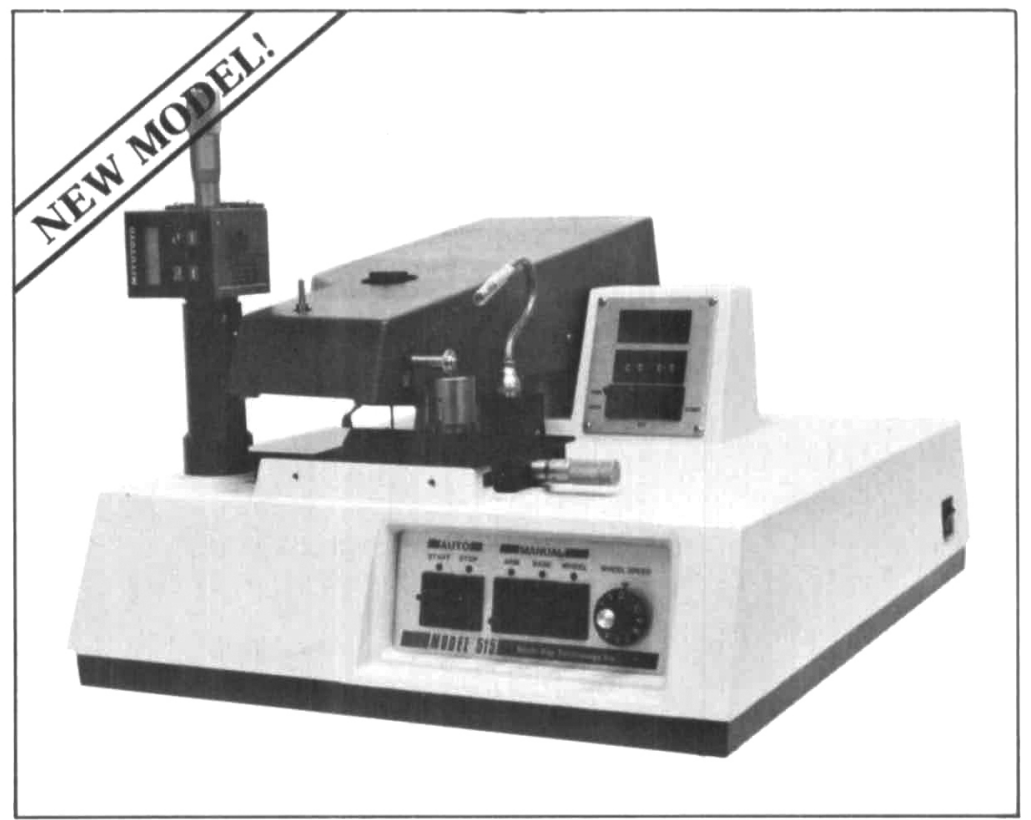

SBT MODEL 515 Precision Dimpling Instrument for TEM Specimen Preparation. requirements for design and performance-time and time again!

- Slicing Instruments

- Lapping \& Polishing Instruments

- Orientation Instruments
- TEM Sample Preparation Instruments

- Wafer Mounting Fixtures

- Sample Preparation Supplies

\section{WHY COMPROMISE?}

\section{SET}

\section{SOUTH BAY}

TECHNOLOGY

inc.

(818) 442.1839

5209 TYLER AVENUE — TEMPLE CITY, CALIFORNIA 91780-3698

TELEX: 311882

Australia/New Zealand

Imbros Pty. Ltd.

9 Warwick Street

G.P.O. Box 917

Hobart, Tasmania 7001

Phone: 34 9899/Telex: $\cdot 58092$

Fax: 344532

\section{France}

Cryophysics, SA

9. Rue Dallery

Jouy-En-Josas, F-78350, France

Phone: 39-56-00-66/Telex: 696096
Austria/West Germany

Cryophysics GmbH

Landwehrstrasse 48

Darmstadt D-6100, West Germany

Phone: (06151) 86281/Telex: 419594

Fax: (6151) 84481

Japan

Meiwa Shoji Co., Ltd.

Tenshin Building

12-14, Kyomachibori 1-Chome

Nishi-Ku, Osaka 550 Japan

Phone: 06-448-3474/Telex: 64849

Fax: 816-447-0074
China

Global Technology

Xiyuan Hotel Room 919

Xijiao, Erligou

Beijing, China

Phone: 890721 Ext. 919

Telex: 22831

United Kingdom

Testbourne Ltd.

9 Sheppard Road

Basingstoke, Hampshire

RG21 3HT, England

Phone: (STD 0256) 467055

Telex: 858029 